\title{
Hypothesis of Memorisation Process
}

\author{
Bern Schmidt $\mathbf{S}^{*}$, Eryn Rekgai ${ }^{2}$ and Muller G Hito ${ }^{2}$ \\ ${ }^{1}$ Department of Fundamental Neuroscience, University of Lausanne, Switzerland \\ ${ }^{2}$ Department of Psychology and Sports Science, Justus-Liebig University, Giessen, Germany
}

Received: 9 November, 2017; Accepted: 29 November, 2017; Published: 7 December, 2017

*Corresponding author: Bern Schmidt, Department of Fundamental Neuroscience, University of Lausanne, Switzerland; Email: s.bern@yahoo.com

\section{Introduction}

The experience of our environment drives our perception and our learning capacity [1-7]. Recent findings demonstrated that, repetitive stimuli influenced the responses of neurons, as well as their learning process [8]. The most common learning mechanism is visual adaptation that allows neurons to acquire new property paralleling learning experience. Following a sine wave grating stimuli acting as reminder, there is a change over time in the response selectivity of neurons of the sensory system to this constant stimulus. It is usually recorded as a change in the preferred stimulus. For instance, for each neuron, the stimulation of its receptive field by a sine-wave grating presented in different orientations makes it possible to determine a preferred orientation; which is the orientation of the grating inducing the highest frequency of the action potentials [8]. However, following a period of adaptation to non-preferred orientation selectivity, the optimal orientation shift to a new value hence the neuron acquires a new selectivity. The displacement of this peak is named shift of orientation. This short-term plasticity process occurs almost immediately after stimulus presentation, within 1 minute; and most of neurons displayed shifts of peaks of oriented tuning curves. In addition to orientation in many animals, similar modifications of optimal stimuli were reported for direction, speed, spatial frequency [9-12].

Neurons exposed to a repeated visual stimulus at brief intervals exhibited weaker responses to the following stimulus in comparison to the initial stimulus. These changes in evoked firing are attributed to the dynamic balance between excitatory neurons and inhibitory interneuron, which interacts continuously to adjust responses to the current stimulus. The common methodology used by researchers, is some visual or auditory stimuli lasting enough to induce modification in the neuronal response. After stimuli, responses are estimate by the changes in the dynamic and the behavior of cortical neuronal responsiveness [12, 13]. Recently several authors reported that, when visual stimulation is made simultaneously with two following stimuli (both with different attributes) influencing the following parameters: variability of the responses (Fano Factor), the orientation selectivity index (OSI), the dynamic of the cell (Bandwidth); responses seemed more prominent [8].
Certainly, stimuli sequence changes the pattern of information coming from visual system, but whom impacts more the responses of neurons during this sequence: That is how the first stimulus influences the response to the following target? Here we hypothesized that, the effects of a first visual experience on responses of neurons left in memory during enough time to influences complex stimulation reported by Etindele Sosso and Al [5, 14-18]. This state of things may be validated with a multidisciplinary methodology, using invasive and non-invasive imaging technics to assess live side effects of synaptic modification. It can also be a way to understand how neurodegeneration appears, and how reverse them for some illnesses.

\section{Conclusion}

Current literature suggested that, a previous experience affects differentially the short-term plasticity of cortical population. Balance excitation-inhibition can me modified during a small window, to changes the functional responses of neurons and the local activity in V1. It is a strong confirmation of underlying mechanism of sensory-induced plasticity, driving by synaptic circuitry from primary visual cortex until brain structure involved in memorisation and encoding.

\section{References}

1. Ernst, C., N. Mechawar, and G. Turecki, Suicide neurobiology. Prog Neurobiol, 2009. 89(4): p. 315-33.

2. Julius B M Anang, Jean-Francois Gagnon, Josie-Anne Bertrand, Silvia Rios Romenets, Veronique Latreille, Michel Panisset. Predictors of dementia in Parkinson disease: a prospective cohort study. Neurology.2014; 83(14):1253-1260. doi: 10.1212/WNL.0000000000000842

3. Baron K G, K J Reid. Circadian misalignment and health. Int Rev Psychiatry.2014;26(2):139-54.doi:10.3109/09540261.2014.911149.

4. Cheng GH, Chan A, Lo JC. Importance of social relationships in the association between sleep duration and cognitive function: data from community-dwelling older Singaporeans. Int Psychogeriatr.2017:15-19.10.1017/S1041610217001041.

5. Etindele Sosso FA. Negative Involvement of the Working Environment in the Occurrence of Cognitive Disorders. Transl Biomed. 2017;8:2. doi:10.2167/2172-0479.1000109 
6. Etindele Sosso FA and S Raouafi, Brain Disorders: Correlation between Cognitive Impairment and Complex Combination. Mental Health in Family Medicine. 2016;12:215-222.

7. Sosso FE, Neurocognitive Game between Risk Factors, Sleep and Suicidal Behaviour. Sleep Science. 2017;10(1):41-46. doi:10.5935/19840063.20170007

8. Etindele Sosso FA, Visual dot interaction with short-term memory. Neurodegener Dis Manag. 2017;7(3):183-190. doi:10.2217/nmt2017-0012.

9. Etindele Sosso F, M Hito, and S Bern, Basic activity of neurons in the dark during somnolence induced by anesthesia. J Neurol Neurosci, 2017;8(3): 203. doi :10.21767/2171-6625.1000203

10. Bertrand JA, Bedetti C, Postuma RB, Monchi O, Génier Marchand D, Jubault T, et al. Color discrimination deficits in Parkinson's disease are related to cognitive impairment and white-matter alterations. Mov Disord, 2012;27(14):1781-1788. doi :10.1002/mds.25272

11. Bertrand JA, McIntosh AR, Postuma RB, Kovacevic N, Latreille V, Panisset $\mathrm{M}$, et al. Brain Connectivity Alterations Are Associated with the Development of Dementia in Parkinson's Disease. Brain Connect. 2016;6(3): 216-224. doi:10.1089/brain.2015.0390.

12. Dubé J, Lafortune M, Bedetti C, Bouchard M, Gagnon JF, Doyon J. et al. Cortical thinning explains changes in sleep slow waves during adulthood. J Neurosci. 2015;35(20):7795-7807. doi :10.1523/ JNEUROSCI.3956-14.2015.
13. Omond S, Ly LMT, Beaton R, Storm JJ, Hale MW, Lesku JA. Inactivity Is Nycthemeral, Endogenously Generated, Homeostatically Regulated, and Melatonin Modulated in a Free-Living Platyhelminth Flatworm. Sleep, 2017.40(10). doi: 10.1093/sleep/zsx124.

14. Etindele Sosso F. Sleep Disorders and Insomnia: Effects on a Young Population. Psychology and Psychiatry, 2017;2:26-32.

15. Etindele Sosso, F. and S. Raouafi, Appropriate Sleep Duration and Physical Activity Modulate Cognitive Improvement. J Sleep Disor: Treat Care 5: 4. J Sleep Disor Treat Care. 2016. 5(4). doi : 10.4172/23259639.1000182

16. Etindele Sosso F A, O Nakamura and N Mitsu. Evaluation of Combined Effects of Insomnia and Stress on Sleep Quality and Sleep Duration. Journal of Neurology and Neuroscience. 2017. 8(3). doi :10.21767/2171-6625.1000202.

17. Raouafi S and F A Etindele Sosso. Cyberpsychology: Video Games as a perspective for Cognitive Training. Ment Health Addict Res. 2017. 2(3):1-2. doi:10.15761/MHAR.1000141.

18. Sosso FAE R S. An Overview of Positive Interaction between Exercise and Mental Health. J Neurol Neurosci. , 2017. 8(4):215-219. 